\title{
Dynamic O-D Demand Estimation: Application of SPSA AD-PI Method in Conjunction with Different Assignment Strategies
}

\author{
Marialisa Nigro $\mathbb{D}^{\mathrm{D}},{ }^{1}$ Akmal Abdelfatah, ${ }^{2}$ Ernesto Cipriani, ${ }^{1}$ Chiara Colombaroni, ${ }^{3}$ \\ Gaetano Fusco, ${ }^{3}$ and Andrea Gemma ${ }^{1}$ \\ ${ }^{1}$ Department of Engineering, Roma Tre University, Rome, Italy \\ ${ }^{2}$ American University of Sharjah, Sharjah, UAE \\ ${ }^{3}$ Department of Civil, Constructional and Environmental Engineering, Sapienza University of Rome, Rome, Italy \\ Correspondence should be addressed to Marialisa Nigro; marialisa.nigro@uniroma3.it
}

Received 31 October 2017; Revised 26 February 2018; Accepted 8 April 2018; Published 23 May 2018

Academic Editor: Luca D’Acierno

Copyright (C) 2018 Marialisa Nigro et al. This is an open access article distributed under the Creative Commons Attribution License, which permits unrestricted use, distribution, and reproduction in any medium, provided the original work is properly cited.

\begin{abstract}
This paper examines the impact of applying dynamic traffic assignment (DTA) and quasi-dynamic traffic assignment (QDTA) models, which apply different route choice approaches (shortest paths based on current travel times, User Equilibrium: UE, and system optimum: SO), on the accuracy of the solution of the offline dynamic demand estimation problem. The evaluation scheme is based on the adoption of a bilevel approach, where the upper level consists of the adjustment of a starting demand using traffic measures and the lower level of the solution of the traffic network assignment problem. The SPSA AD-PI (Simultaneous Perturbation Stochastic Approximation Asymmetric Design Polynomial Interpolation) is adopted as a solution algorithm. A comparative analysis is conducted on a test network and the results highlight the importance of route choice model and information for the stability and the quality of the offline dynamic demand estimations.
\end{abstract}

\section{Introduction}

Dynamic Traffic Assignment (DTA) models are among the most effective tools for analysis and prediction of traffic conditions, especially in congested road networks. To provide accurate and reliable estimates, DTA models need information on the distribution of the trips in space and time (dynamic demand matrices) that are assigned to the network. It is straightforward that a better estimation of the dynamic demand matrices leads to a better estimation and prediction of traffic conditions.

This paper considers the offline estimation of the dynamic origin-destination $(\mathrm{O}-\mathrm{D})$ demand matrices as a starting point that can be upgraded to deal with real-time information for online demand estimation. The offline estimate of the dynamic demand matrices assumes a starting demand value to be known based on the available information on traffic conditions on the network. This is a highly undetermined, nonlinear, nonconvex problem, which was the object of a relevant research effort in the last years [1].
The offline dynamic estimation problem is usually approached as a bilevel problem. The upper-level problem consists of the adjustment of a starting demand using traffic measures, which are in turn linked to the dynamic demand. This link is generated from the dynamic traffic network assignment problem at the lower level, solved by using a dynamic traffic assignment (DTA) model.

Cascetta et al. [2] approached the dynamic O-D matrix estimation problem by introducing two different estimators: simultaneous and sequential. The former estimates all the matrices for all time slices using the whole set of traffic counts; the latter estimates at each step the matrix for a given time slice expressed as a function of the traffic counts within the same time slice and the already estimated previous demand matrices. The simultaneous estimator is more robust and provides better estimates than the sequential one. However, it requires knowledge of the dynamic assignment matrix, which has huge dimensions in real networks, and is computationally very expensive. On the other hand, Marzano et al. [3] pointed 
out that the highly indeterminacy of the problem is often cause of poor performances of the solution.

The research effort was directed mainly to improve the efficiency and the effectiveness of the solution methods, by following different research lines [4]: (i) introducing some approximation to the optimization method to reduce the computational effort; (ii) including more variables exploiting all available sources of information on traffic performances; (iii) assuming some simplification into the traffic assignment model.

As far as the first line, Yang [5] provided two heuristic solution approaches: iterative estimation-assignment and sensitivity-analysis based algorithms. The iterative approach does not guarantee the convergence to the solution. The iterative approach is theoretically not fully satisfactory, because the upper-level problem neglects the dependence of link flows on O-D matrix. On the other hand, sensitivityanalysis algorithm needs to approximate the derivatives through simulation for each O-D pair and each time interval at every iteration. Balakrishna and Koutsopoulos [6] introduced gradient approximation methods within a simultaneous perturbation stochastic approximation (SPSA) framework in order to reduce the number of simulation runs when calculating numerical derivatives or gradients. Cipriani et al. [7] proposed some modifications of the basic SPSA, introducing the Asymmetric Design (AD) for gradient computation and the Polynomial Interpolation (PI) of the objective function along the gradient direction. One of the recent contributions, related to the bilevel approach, is to try to jointly solve the offline demand estimation with the user equilibrium (UE) DTA problem [8]. In the same year, Djukic et al. [9] applied the principal component analysis method to reduce the O-D demand variables. Then, Cantelmo et al. [11] extended the SPSA AD-PI method to a second-order approximation by applying a Quasi-Newton method. In addition, they proposed an adaptive approach that computes, at each iteration, the weights in the gradient computation according to the relevance of any $\mathrm{O}-\mathrm{D}$ pair as computed. Lu et al. [12] introduced an enhanced SPSA algorithm, which incorporates spatial and temporal correlation between parameters and measurements to minimize the noise generated by uncorrelated measurements and reduce the gradient approximation error. While in the original SPSA the objective function is a single scalar, in the weighted SPSA it is a vector, whose gradient components are weighed by a matrix that expresses the correlations between parameters and measurements. Numerical tests to a small size and a real-size road network highlighted that the enhanced method improved the efficiency and the accuracy of the original SPSA estimation method.

As far as the second research line, considerable attention has been given to the role of different traffic measures adopted inside the $\mathrm{O}-\mathrm{D}$ estimation procedure, for offline and online applications in addition to the usually adopted link counts, specifically speed and link occupancy [7, 8, 13], probe data from vehicle equipped by AVI tags [14-21]; aggregate demand data, such as traffic emissions and attractions by zones [7, 22, 23]; measure link speeds and path travel times [24].
As far as the third research line, Frederix et al. [25] provided a linear approximation of the relationship between O-D flows and link flows, based on a marginal computation method that performs a perturbation analysis using the kinematic wave theory. Lu et al. [26] presented a singlelevel nonlinear path flow-based optimization model, which does not require explicit dynamic link-path incidences and applies a Dynamic Network Loading model based on Newell's simplified kinematic wave theory in the DUE assignment process. Cascetta et al. [27] proposed a "quasi-dynamic" framework for estimation of O-D flows, in which $\mathrm{O}-\mathrm{D}$ shares are assumed constant across a reference period, while total flows leaving each origin are assumed varying for each subperiod within the reference period. Cipriani et al. [28] applied a quasi-dynamic traffic assignment model (QDTA [29]) that approximates the dynamic traffic model by steadystate intervals and applies approximate performance functions in order to reduce the computational burden to solve the estimation problem. Cantelmo et al. (2016) proposed a utility-based formulation for the demand estimation, which is able to incorporate activity duration and to consider different activity patterns. The building block of this methodology is to adopt a utility-based departure time choice model in the DTA and to exploit this model to derive the demand temporal distribution.

The research illustrated in this paper finds its starting motivations just in the results obtained by the contribution of Cipriani et al. [28], where the approximation of the DTA with the QDTA model to solve the O-D estimation problem was able to generate interesting results in terms of traffic measures reproduction. Specifically, the SPSA AD-PI algorithm was adopted in conjunction with the QDTA on a subnetwork of the city of Rome, consisting of 113 traffic zones, 757 links, and 335 nodes, where the traffic measures, in order to adjust the starting value of the demand, were true data of flows and speeds on 41 links of the network. These true data have been derived by Floating Car Data (FCD) collected during a national project (Pegasus Project) for the whole metropolitan area of Rome: a fleet of 103,000 floating vehicles, travelling 9 million trips, provided 104 million records containing positions and speeds during one month (May 2010). About 80,000 vehicles of these 103,000 floating vehicles crossed the study area, thus generating a huge amount of data with high disaggregation. Simulated link data, at the end of the O-D estimation, resulted in a high correspondence with the true data, obtaining improvement of approximately $65 \%$ in terms of relative mean error with respect to the starting conditions.

Thus, on one hand these results seem to show that there is the challenge to approximate DTA models, reducing both the calibration efforts and the computation time and laying the foundation for applications to time-dependent $\mathrm{O}$ $\mathrm{D}$ estimation problems on real-size networks; on the other hand, there is a question worth of investigation, that is, the degree of approximation that can be introduced by using the QDTA to simulate the user's behaviours.

Such considerations have to be integrated into a recent analysis of a large dataset of FCD collected in Rome that highlights that users moving from the same origin at the same time interval (or with quite close departure times) choose 
multiple routes with different observed travel times to reach the same destination $[30,31]$. The finding of different actual route travel times experienced by drivers within the same time interval raises some issues related to the concept of equilibrium in the dynamic traffic assignment phase.

The first issue concerns the realism of the behavioural assumptions underlying the dynamic assignment models: dynamic equilibrium traffic models provide detailed information regarding the temporal profile of performance metrics (travel times, speeds, and densities), extend the equilibrium concept introduced in the static model, and then assume the same assumptions that drivers are rational and have perfect information on network conditions they will face approaching their destinations. Whether the real route choice mechanism should be based on instantaneous rather than on experienced travel times (or a combination of them) is still an open research topic [32]. Even if dynamic equilibrium principles are often referred to experienced travel times, loading vehicles on the network according to instantaneous travel times reflects the fact that either drivers choose their shortest routes on the basis of an instantaneous picture of the network (conditions) taken at their departure time, as more and more frequently now happens, through websites visited at home (pretrip information), or they adjust their route while travelling, on the basis of information received on real-time updated mobile devices while on the route (en route information).

The dispute between instantaneous and experienced travel times is strongly related to the second issue that raises from FCD observations: the convergence of assignment procedures. As it is well known, the procedure is considered to have converged, approaching equilibrium conditions, when no simulated driver can improve his travel time by shifting to an alternative route; thus, no change in experienced travel times can be detected and no change in traffic pattern occurs on the network, even if running additional iterations. This implies that the network has reached a condition of stability, which is the third issue, one of the major interests for this study and has motivated the present paper. Stability of the equilibrium condition is reflected by the algorithm progression as the condition of detecting no change (or negligible change) in network conditions when running additional iterations after the equilibrium has been reached. This condition can be alternatively seen from the supply side by producing a minor change to the network features, for instance, by changing the speed limit on a link and detecting only local changes of traffic conditions or, from the demand side, by slightly changing the demand, for instance, adding one vehicle to an $\mathrm{O}-\mathrm{D}$ pair, and detecting only negligible changes of traffic flow patterns.

The latter example is of great relevance in the present paper because it affects the shape of the objective function being minimized in the demand estimation problem: if the traffic assignment is not stable then the objective function is very noisy and, moreover, may exhibit no descent direction towards the real demand matrix.

Such observations have motivated the investigation of the paper, which is to solve the demand estimation problem under different traffic assignment conditions and criteria, also adopting approximation of DTA models, thus investigating the impact of different route choice modeling on the convergence and the accuracy of the estimation. The offline dynamic demand estimation problem has been solved on a test network with the adoption of a bilevel approach based on the SPSA AD-PI algorithm. Traffic assignments required at the lower level have been performed by using the QDTA model [29] and different route choice options given by the Dynasmart model [33-37].

The paper is organized in four sections including this introduction. Section 2 deals with the offline dynamic O$\mathrm{D}$ matrices estimation (DODME) problem: it describes the main concepts behind the DTA models adopted in the study and the solution approach based on the SPSA AD-PI method. Section 3 presents the results of numerical tests carried out by applying the solution method to a test network in combination with different kinds of traffic measurements and different assignment models. Conclusions follow, in Section 4.

\section{Problem Formulation}

This formulation considers a network consisting of a set of $\operatorname{arcs}(\mathbf{A})$, a set of nodes $(\mathbf{N})$, and a set of routes $(\mathbf{R})$. Given the period of analysis $T$, divided into $H$ intervals, a subset of links $\mathbf{L}=\left\{a_{1}, a_{2}, \ldots, a_{\mathrm{L}}\right\} \subset \mathbf{A}$ and nodes $\mathbf{Q}=\left\{n_{1}, n_{2}, \ldots, n_{\mathbf{Q}}\right\} \subset$ $\mathbf{N}$ equipped with sensors, a subset of monitored routes $\mathbf{P}=\left\{r_{1}, r_{2}, \ldots, r_{\mathrm{P}}\right\} \quad \subset \mathbf{R}$, and a set of centroids $\mathbf{C}=$ $\left\{c_{1}, c_{2}, \ldots, c_{\mathrm{D}}\right\}$, where the demand is assumed to have origin and destination, the problem of offline simultaneous dynamic demand estimation with multiple sources of information can be formulated as follows:

$$
\begin{aligned}
& \left(\mathbf{d}_{1}^{*}, \ldots, \mathbf{d}_{H}^{*}\right)=\underset{\left(\mathbf{x}_{1}, \ldots, \mathbf{x}_{H}\right)}{\operatorname{argmin}}\left[f_{1}\left(\mathbf{x}_{1}, \ldots, \mathbf{x}_{H}, \mathbf{d}_{1}, \ldots, \mathbf{d}_{H}\right)\right. \\
& \quad+\sum_{l} f_{2}^{l}\left(\mathbf{y}_{1}, \ldots, \mathbf{y}_{H}, \widehat{\mathbf{y}}_{1}, \ldots, \widehat{\mathbf{y}}_{H}\right) \\
& \quad+\sum_{q} f_{3}^{q}\left(\mathbf{z}_{1}, \ldots, \mathbf{z}_{H}, \widehat{\mathbf{z}}_{1}, \ldots, \widehat{\mathbf{z}}_{H}\right) \\
& \left.\quad+\sum_{p} f_{4}^{p}\left(\mathbf{w}_{1}, \ldots, \mathbf{w}_{H}, \widehat{\mathbf{w}}_{1}, \ldots, \widehat{\mathbf{w}}_{H}\right)\right]
\end{aligned}
$$

where

$$
\begin{aligned}
& \mathbf{x}_{i}=\text { estimated } \mathrm{O}-\mathrm{D} \text { matrix for departing time interval } \\
& i, i=1 \cdots H ; \\
& \mathbf{d}_{i}=\text { seed } \mathrm{O}-\mathrm{D} \text { matrix for departing time interval } i \\
& i=1 \cdots H ; \\
& \mathbf{y}_{i}=\text { simulated values on subset of links } \mathbf{L} \text { in the time } \\
& \text { interval } i, i=1 \cdots H ; \\
& \widehat{\mathbf{y}}_{i}=\text { collected measures on subset of links } \mathbf{L} \text { in the time } \\
& \text { interval } i, i=1 \cdots H ; \\
& l=\text { type of collected measures on links } \mathbf{L} ; \\
& \mathbf{z}_{i}=\text { simulated values on subset of nodes } \mathbf{Q} \text { in the time } \\
& \text { interval } i, i=1 \cdots H ;
\end{aligned}
$$




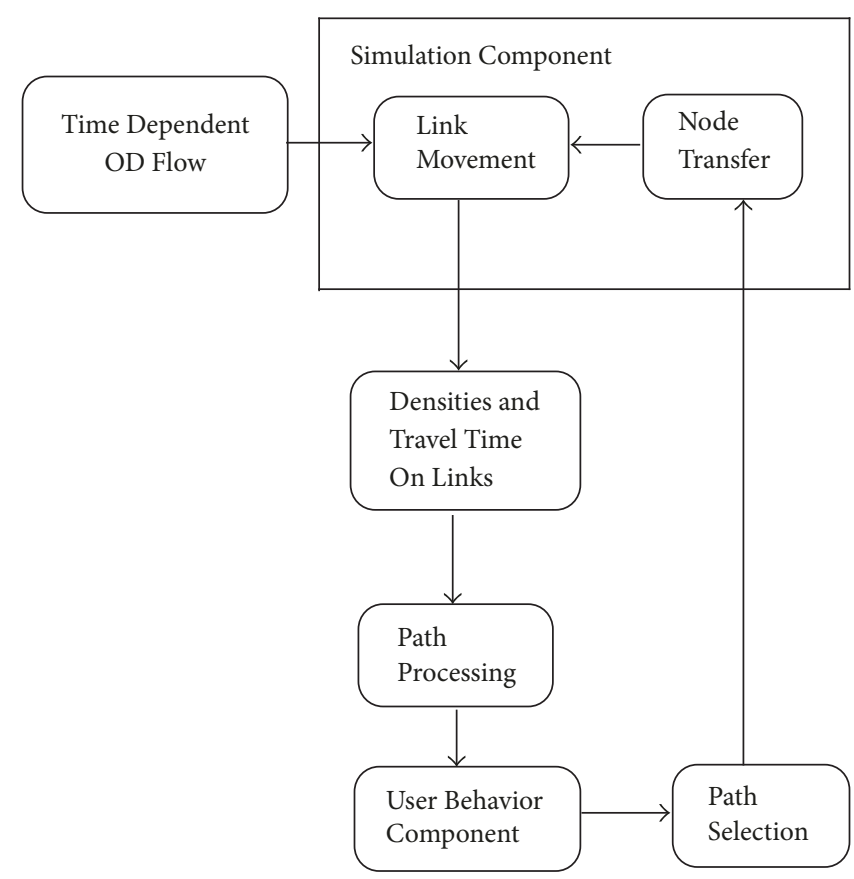

FIGURE 1: Dynasmart modeling framework [40].

$\widehat{\mathbf{z}}_{i}=$ collected measures on subset of nodes $\mathbf{Q}$ in the time interval $i, i=1 \cdots H$;

$q$ = type of collected measures on nodes $\mathbf{Q}$;

$\mathbf{w}_{i}=$ simulated values on subset of routes $\mathbf{P}$ for departing time interval $i, i=1 \cdots H$;

$\widehat{\mathbf{w}}_{i}=$ collected measures on subset of routes $\mathbf{P}$ for departing time interval $i, i=1 \cdots H$;

$p=$ type of collected measures on routes $\mathbf{P}$.

Functions $f_{1}, f_{2}^{l}, f_{3}^{q}$, and $f_{4}^{p}$ are goodness of fit functions related to different kinds of information about demand and traffic patterns that in general may be available. If a reliable prior O-D matrix (called "seed matrix") is available, the objective function to be minimized can suitably include function $f_{d}$ as the distance with respect to the seed matrix. This specification of the estimation problem is usually referred to as demand adjustment in literature. Moreover, the formulation reported in (1) permits the introduction of different types of data collected on the network in order to better reproduce the demand in space and time. Examples of such types of data are as follows: (i) measures on links as flow, speed, occupancy, queue length, and density; (ii) measures on nodes, for example, turning movements; (iii) measures on routes as travel time, distance travelled, or path flow fractions. In a dynamic framework, the relationship between the values of the measured variables and the demand is captured by simulation is presented as

$$
\begin{array}{rr}
\mathbf{y}_{1}, \ldots, \mathbf{y}_{H}=\Gamma\left(\mathbf{x}_{1}, \ldots, \mathbf{x}_{H}\right) & \forall l \\
\mathbf{z}_{1}, \ldots, \mathbf{z}_{H}=\Gamma\left(\mathbf{x}_{1}, \ldots, \mathbf{x}_{H}\right) & \forall q \\
\mathbf{w}_{1}, \ldots, \mathbf{w}_{H}=\Gamma\left(\mathbf{x}_{1}, \ldots, \mathbf{x}_{H}\right) & \forall p .
\end{array}
$$

Operator $\Gamma$ is a generic traffic assignment model able to represent, given a dynamic demand as an input, the time variation of the measures along the different elements of the network. Generally $\Gamma$ is reported in previous works as a Dynamic User Equilibrium Traffic Assignment (DTA-DUE); however similar approaches in literature adopt also Dynamic Network Loading (DTA-DNL), usually when there is no need for route choice $[10,38]$. Nonequilibrium approaches, such as system optimum approach (DTA-SO) or quasi-dynamic traffic assignment (QDTA), can be also adopted. It is easy to assume that the choice of $\Gamma$ can influence the results of the demand estimation, which is the focus of this paper.

To apply different assignment strategies and simulate different behavioural assumptions on route choice, the stateof-the-art DTA software Dynasmart (DYnamic Network Assignment Simulation Model for Advanced Road Telematics) is used [39]. This is a simulation assignment model that integrates traffic flow models, path processing methodologies, behavioural rules, and information supply strategies. The traffic model is a mesoscopic simulation model, which applies a macroscopic concept for moving the vehicles on the links. For the movement of vehicles at intersections, Dynasmart adopts a microscopic simulation concept. The modeling framework for Dynasmart is presented in Figure 1. The path selection process may apply any of the following rules: pretrip information, en route real-time information, Dynamic User Equilibrium (DUE), and system optimum (SO).

Drivers' behaviour in response to en route real-time information is simulated according to the bounded rationality approach, which assumes that drivers will change their path according to travel time information received only if the new path yields travel time savings greater than given thresholds. 
About the possibility of approximating the DTA, the QDTA approach by Fusco et al. [29] has been adopted. The QDTA model assumes that steady-state conditions holding in each time slice in which the simulation interval is divided. The great advantage of this strong assumption is that it exploits link-cost functions used in static equilibrium models that are applied in the most practical applications. Thus, the calibration effort is much lower than that required by the more complex dynamic traffic assignment models; however, a validation performed on a large set of Floating Car Data provided error statistics close to those obtained in one of the most advanced applications of a dynamic simulation traffic assignment model reported in literature.

The dynamics of traffic is introduced in the network loading process, which simulates the progression of all packets of flow in each time interval by moving them, according to the value of speed corresponding to the link flow, up to the position on the network reached in the time interval. Because of the link-cost functions used, the model does not simulate the processes of queue progression and clearance but computes anyway the traffic congestion in terms of link travel time.

It is assumed that users do not modify their route choice during the travel; thus, at each iteration, routes are computed only for the new users that enter the network. However, because of the time-dependent interaction of flows on the network, origin-destination (O-D) flows starting at a generic time interval may be affected by flows starting at a successive time interval and overlapping their route (consequently changing their travel time).

Since Dynasmart is assumed as term of reference in this laboratory application, the QDTA model has been calibrated by applying a Particle Swarm Optimization algorithm to determine the parameters of volume-delay functions that better approximate the results provided by Dynasmart on the test network used in this experiment.

2.1. Solution Approach. In the following, the main principles of SPSA AD-PI, adopted in this paper as the solution method for the offline simultaneous dynamic demand estimation, are summarized. SPSA AD-PI was firstly proposed by Cipriani et al. in 2010 and it is based on the path search optimization method of Spall, [41, 42]. It works with a gradient approximation, thus limiting the computational resources required for the calculation of a standard numerical gradient.

Considering the generic iteration $k+1$ of the algorithm, the dynamic O-D matrix $\mathbf{x}_{k+1}$ can be computed as

$$
\mathbf{x}_{k+1}=\mathbf{x}_{k}-a_{k} \overline{\overline{\mathbf{g}}}\left(\mathbf{x}_{k}\right),
$$

where

$\mathbf{x}_{k}=$ estimated dynamic O-D matrix for iteration $k$;

$a_{k}=$ gain sequence at iteration $k$ of the O-D estimation algorithm;

$\overline{\widehat{\mathbf{g}}}\left(\mathbf{x}_{k}\right)=$ average approximated gradient at iteration $k$.
The average approximated gradient $\overline{\widehat{\mathbf{g}}}\left(\mathbf{x}_{k}\right)$ is calculated as the average of $m$ gradient approximations at iteration $k$ :

$$
\overline{\mathbf{g}}\left(\mathbf{x}_{k}\right)=\text { average }_{m}\left(\widehat{\mathbf{g}}_{m}\left(\mathbf{x}_{k}\right)\right) .
$$

Each gradient approximation $\widehat{\mathbf{g}}_{m}\left(\mathbf{x}_{k}\right)$ is based on a simultaneous perturbation of each component of $\mathbf{x}_{k}$; thus, all elements of $\mathbf{x}_{k}$ are randomly perturbed together adopting a random perturbation vector $\Delta_{m}$ to obtain one additional point with respect to $\mathbf{x}_{k}$ (Asymmetric Design: $\mathrm{AD}$ ) for which the objective function $z$ is evaluated:

$$
\begin{aligned}
\widehat{\mathbf{g}}_{m} & \left(\left(\mathbf{x}_{1} \cdots \mathbf{x}_{\mathbf{n}_{\mathbf{h}}}\right)_{k}\right) \\
= & \frac{z\left(\left(\mathbf{x}_{1} \cdots \mathbf{x}_{\mathbf{n}_{\mathbf{h}}}\right)_{k}+c_{k} \Delta_{m}\right)-z\left(\left(\mathbf{x}_{1} \cdots \mathbf{x}_{\mathbf{n}_{\mathbf{h}}}\right)_{k}\right)}{c_{k}} \\
& \cdot\left[\begin{array}{c}
\left(\Delta_{m}^{1}\right)^{-1} \\
\cdot \\
\left(\Delta_{m}^{n v}\right)^{-1}
\end{array}\right]
\end{aligned}
$$

with

$z=$ objective function (OF) as defined in (1), where the simulated traffic measurements are obtained directly by performing the assignment process with Dynasmart (i.e., it is an assignment matrix free method).

$\Delta_{m}=n_{v}$-dimensional random perturbation vector (with $n_{v}=H \times D^{2}$ ); the distribution of $\Delta$ has to comply the condition that the $\left\{\Delta_{m}^{j}\right\}$ elements of the perturbation vector are independent and symmetrically distributed around 0 with finite inverse moments $E\left(\left|\Delta_{m}^{j}\right|^{-1}\right)$ for all $m, j$.

$c_{k}=$ the step to compute the approximated gradient.

The linear optimization in (3) is solved using a Polynomial Interpolation (PI) of the objective function: from the solution $\mathbf{x}_{k}$ at iteration $k$, other three possible dynamic demand matrices $\left(\mathbf{x}_{k 1}, \mathbf{x}_{k 2}, \mathbf{x}_{k 3}\right)$ are computed along the descendent direction founded by the average approximated gradient (Figure 2(a)). Then, the corresponding values of the objective function (OFs) are evaluated and the third-degree polynomial function interpolating these points is analytically derived (Figure 2(b)). The minimum point of the Polynomial Interpolation is finally computed and considered as the suboptimal solution of the problem.

About the computational times of SPSA AD-PI, these are function of the number of traffic assignments. Each time the SPSA computes a gradient approximation (5), there is the need to run an assignment by Dynasmart. Since $m$ computations of the gradient approximation are required at each iteration of SPSA (4), the parameter $m$ strongly affects the computational times. It is demonstrated that the increase in the value of $m$ could permit a higher efficiency in terms of iterations of the algorithm; however, this added value is not always able to compensate for the longer computational times per iteration. A value of $m$ equal to $10 \div 15 \%$ of the number of variables can be a good compromise between reliability of the solution and computational times [11]. 


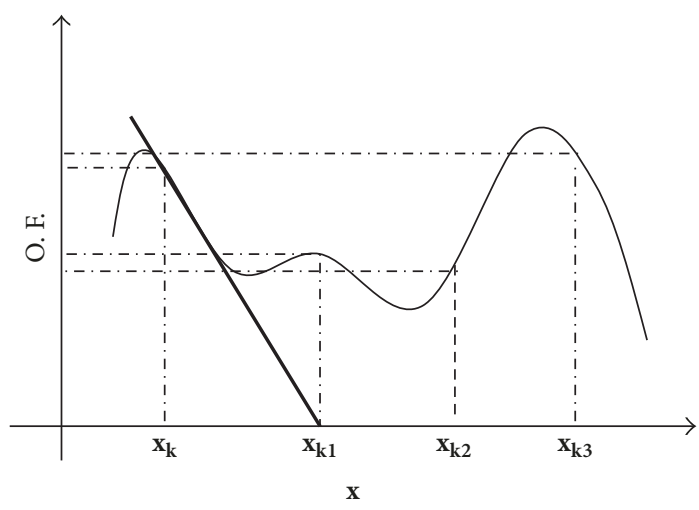

Descendent direction

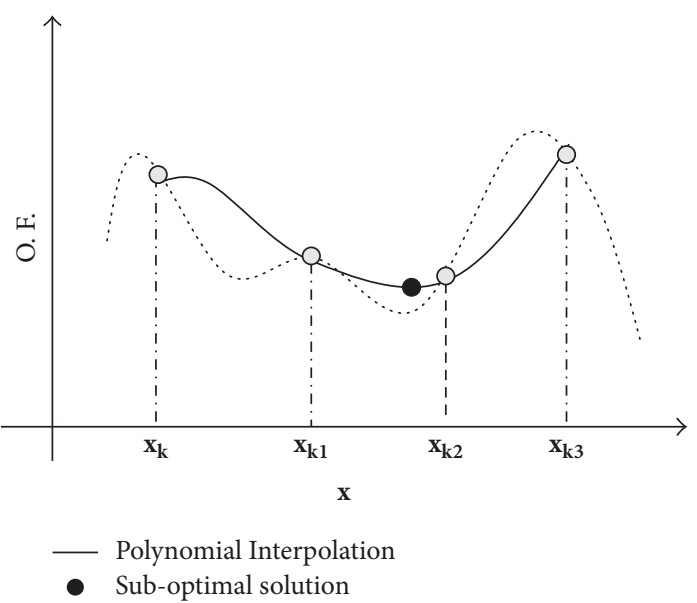

(b)

(a)

Figure 2: One-dimension Polynomial Interpolation (PI).

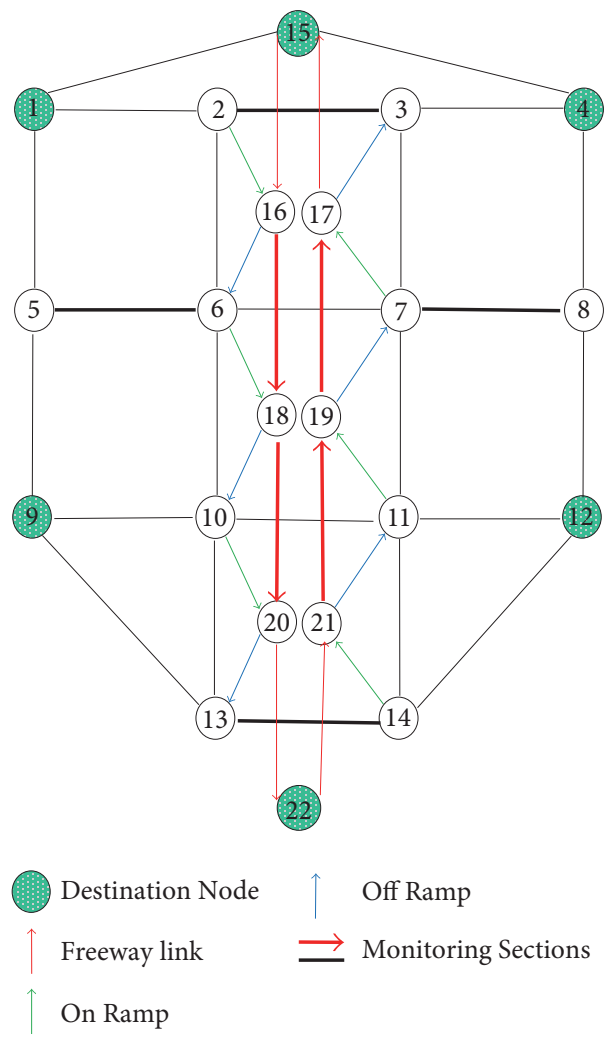

FIGURE 3: Test network.

\section{Numerical Experiments}

Several experiments have been conducted on a test network consisting of 22 nodes (14 are signalized intersections), 68 links (composed by highway links, freeway links, onramp, and offramp links), 6 traffic zones, and a whole planning horizon of 35 minutes discretized into 5 minutes intervals (Figure 3), generating a total value of $252 \mathrm{O}-\mathrm{D}$ variables to be estimated.
In total, 12 links are monitored and monitoring sections are located between the following pairs of nodes: $\{(2,3) ;(5,6)$; $(7,8) ;(13,14) ;(16,18) ;(19,17) ;(18,20) ;(21,19)\}$.

All the experiments start from a traffic demand supposed as the "true" demand, which is assigned on the network in order to procure the measures to be included in the $\mathrm{OF}$ (ground truth conditions). Then, the "true" demand has been perturbed in order to obtain a "seed" demand, which is the starting point for the optimization. The OF in (1) 
TABLE 1: Experiment design.

\begin{tabular}{lcc}
\hline Experiments & Type of DTA & Measures inside the OF for each type of DTA \\
\hline Set I & DUE & OF1: link volume \\
Set II & SO & OF2: link volume + speed \\
Set III & DNL & OF3: link volume + density \\
Set IV & $50 \%$ DUE $-50 \%$ SO & OF4: link volume + queue length \\
Set V & DNL + fixed DUE paths & OF5: link volume + speed + density \\
Set VI & DNL + fixed DUE paths (highest O-D flows) & OF6: link volume + speed + queue length \\
Set VII & DUE + fixed DUE paths (highest O-D flows) & OF7: link volume + density + queue length \\
& & OF8: link volume + density + speed + queue length \\
Set VIII & QDTA & O.F.1: link volume \\
& & O.F.2: link volume + speed \\
& & O.F.3: link volume + density
\end{tabular}

is expressed by the Normalized Root Mean Square Error statistic (NRMSE) as goodness of fit between simulated values and traffic measures.

Specifically, only collected measures on links have been adopted inside the OF (link volume, speed, density, and queue length). Various combinations of types of information are assumed in the different tests, as reported in Table 1.

It is worth noting that no term containing information on prior $\mathrm{O}-\mathrm{D}$ matrix has been included in any OF in order to exploit network measurements in the estimation procedure as much as possible, avoiding anchoring it to the initial estimate. Thus, the goal of the experiments is to investigate the influence of different components of information and different assignment models on the O-D matrix estimation. In fact, strong differences are imposed, both in the total value of the trips (12,638 seed versus 18,900 true) and in the O-D distribution between true and seed.

Different $\Gamma$ operators in (2) have been applied in the different sets of experiments in order to represent different network conditions as resulting from different combinations of user route choices; these are reported in Table 1 by type of DTA: other than the well-known DTA-DNL, DUE, and SO criteria, a set of experiments has been conducted considering one-half of users following the User Equilibrium criterion and the remaining a system optimum criterion (Set IV: $50 \%$ DUE $+50 \%$ SO). From Set V to Set VII, the usually adopted DNL or DUE approaches have been mixed with fixed route choices for a subset of O-D pairs: in Set V some fixed paths (specifically, 28 paths) have been randomly selected from the paths used under DUE conditions. In Sets VI and VII, the fixed paths still belong to the DUE paths but they are selected among the set of $\mathrm{O}-\mathrm{D}$ pairs with the highest flows in order to cover at least the $33 \%$ of the total demand. Finally, in Set VIII, the QDTA has been applied.

As a whole, eight sets of experiments have been conducted, each set following a specific DTA approach and containing a total number of eight types of analyzed OF for the first seven types of DTA criteria and four types of OF for the last QDTA criteria.

All the parameters to be defined for the application of SPSA AD-PI have been chosen according to the directions given in Cantelmo et al. [11]: in particular, parameter $s_{k}$ of (5)
TABLE 2: Efficacy of the O-D estimation method in terms of OF reduction.

\begin{tabular}{lcc}
\hline Experiments & OF reduction [\%] & $\begin{array}{c}\text { Standard deviation } \\
{[\%]}\end{array}$ \\
\hline Set I & -99.58 & 0.19 \\
Set II & -92.20 & 21 \\
Set III & - & - \\
Set IV & -96.12 & 8 \\
Set V & -67.53 & 30 \\
Set VI & -75.88 & 28 \\
Set VII & -90.97 & 24 \\
Set VIII & -73.17 & 14 \\
\hline
\end{tabular}

has been set equal to $2 \%$ from the analysis of the OFs in the neighborhood of the seed matrix.

3.1. Evaluation of SPSA AD-PI and Traffic Measures Reproduction. First comments on the results are related to the efficacy of the SPSA AD-PI in terms of the average OF reduction achieved in each set of tests and the related standard deviation of OF reduction obtained by applying different OFs specifications (Table 2). Results show that the dynamic O-D estimation method is able to work on the different OFs with very high improvement with respect to the starting point. In fact, reductions range from $-67.53 \%$ to $-99.58 \%$ and smaller reductions, although important, are obtained for Set V and Set VI (resp., $-67.53 \%$ and $-75.88 \%$, Table 2), that is, when there is a mix between DNL and fixed DUE paths. Results obtained introducing the QDTA (Set VIII) are comparable with that obtained by using Dynasmart with DNL mixed with fixed DUE paths (Set VI), since QDTA applies a probabilistic statistic network loading. Only Set III of tests, in which the assignment criterion assumed was DNL, produced no OF reduction. The reason for that is explained in the following.

Figure 4 shows why the DNL assignment criterion affects $\mathrm{O}-\mathrm{D}$ matrix estimation negatively. In the figure, a monodimensional scan of the OF1 (i.e., the specification of the OF having only the link volume term) is performed using a 


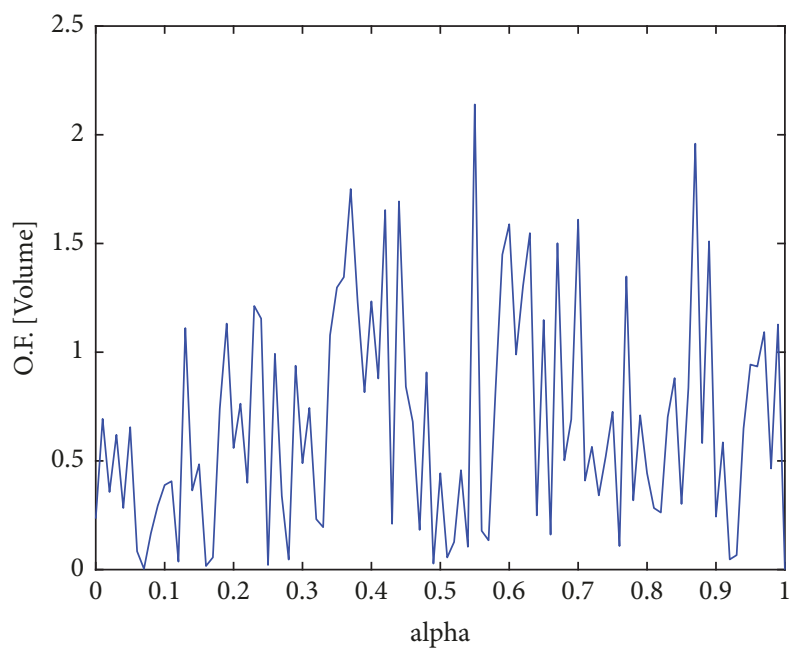

Figure 4: OF1 scan for DTA-DNL between the seed matrix $($ alpha $=0)$ and the true matrix $($ alpha $=1)$.

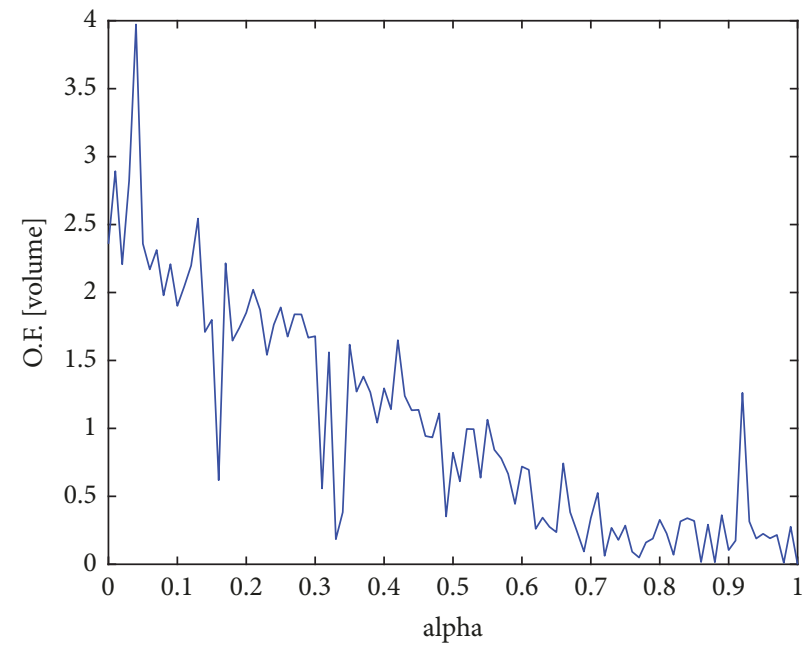

Figure 5: OF1 scan for DTA-DUE.

convex combination between the seed matrix ( $\alpha=0$ in the figure) and the true matrix ( $\alpha=1$ in the figure) and assuming the DNL assignment criterion.

A similar picture is shown in Figure 5, where the DTADUE criterion adopted for Set I is applied.

It is clear that the DNL (Set III) prevents the algorithm from finding a descent direction for the dynamic O-D estimation problem, thus making the SPSA ineffective. This is due to the strong variations of the route choice generated by the dynamic shortest path algorithm when changing the demand matrix. However, scanning the OF1 when the DTA-DUE criterion is applied clearly individuates a descent direction, although with some noise. Similar OF trends can be recorded also for DTA-SO or mixing DUE and SO (resp., Set II and IV).

The reductions of the OFs have a direct impact on the reproduction of the traffic measures: Table 3 reports the average reduction of the difference between link measures and simulated ones obtained from the starting point (seed matrix) to the final point (estimated matrix) and the standard deviations resulting from different OFs, that is, different assignment criteria. In the case of the Dynasmart simulator, not only the measures adopted inside the OFs are reported in the table (i.e., link volume, speed, density, and queue length) but also the other measures that are never used during the $\mathrm{O}-\mathrm{D}$ estimation, as the outflow and the left-turn movements from each monitored link. These measures, although achievable as the output of the simulation with Dynasmart, have been excluded in the process of OFs definition, since they generate a strong noise of the OF as shown, for example, in Figure 6.

The experiments show good results in terms of traffic measure reproduction, both directly (when measures are inside the OF) and indirectly (when measures are not considered in the OF), except for the left-turn movements. For this measure, improvements with respect to the starting point are quite limited (at most about $47 \%$, Set IV, Table 3 ); in some cases, specifically when fixing the DUE paths with the highest 
TABLE 3: Efficacy of the O-D estimation method in terms of reduction of the errors in measures reproductions.

\begin{tabular}{|c|c|c|c|c|c|c|}
\hline Experiments & Link volume & Speed & Density & Outflow & Left-turn movements & Queue length \\
\hline \multicolumn{7}{|l|}{ Set I } \\
\hline Average reduction [\%] & -99.58 & -93.42 & -99.49 & -68.45 & -3.03 & -96.86 \\
\hline St.dev. [\%] & 0.52 & 6.69 & 0.50 & 16.16 & 81.63 & 3.23 \\
\hline \multicolumn{7}{|l|}{ Set II } \\
\hline Average reduction [\%] & -92.32 & -89.25 & -92.24 & -79.06 & -29.96 & -89.85 \\
\hline st.dev. [\%] & 20.97 & 23.74 & 20.50 & 22.71 & 66.86 & 23.50 \\
\hline \multicolumn{7}{|l|}{ Set IV } \\
\hline Average reduction [\%] & -96.61 & -90.55 & -96.44 & -59.21 & -47.45 & -92.67 \\
\hline St.dev. [\%] & 7.02 & 12.41 & 8.50 & 37.34 & 34.77 & 13.23 \\
\hline \multicolumn{7}{|l|}{ Set V } \\
\hline Average reduction [\%] & -67.79 & -65.98 & -66.44 & -37.92 & -8.38 & -66.72 \\
\hline st.dev. [\%] & 29.76 & 29.72 & 20.50 & 35.37 & 119.26 & 30.05 \\
\hline \multicolumn{7}{|l|}{ Set VI } \\
\hline Average reduction [\%] & -76.18 & -74.28 & -76.04 & -63.54 & 128.45 & -74.36 \\
\hline St.dev. [\%] & 27.55 & 27.96 & 25.40 & 29.16 & 130.96 & 28.16 \\
\hline \multicolumn{7}{|l|}{ Set VII } \\
\hline Average reduction [\%] & -91.21 & -90.38 & -90.24 & -82.24 & 568.03 & -90.31 \\
\hline St.dev. [\%] & 24.27 & 23.14 & 22.45 & 15.99 & 446.03 & 21.72 \\
\hline \multicolumn{7}{|l|}{ Set VIII } \\
\hline Average reduction [\%] & -73.59 & -78.85 & -71.03 & - & - & - \\
\hline St.dev. [\%] & 14.81 & 12.48 & 15.30 & - & - & - \\
\hline
\end{tabular}

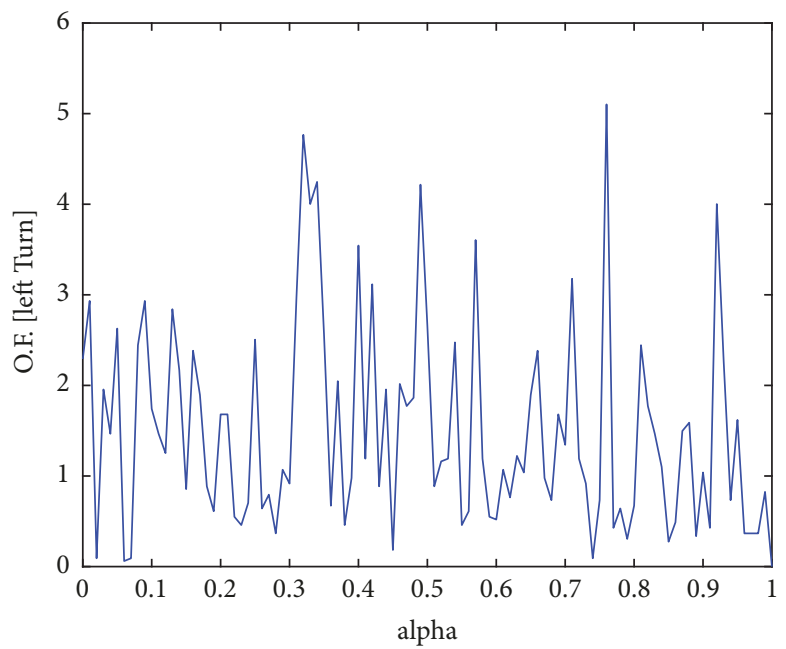

FIGURE 6: Left-turn error index scan for DTA-DUE.

OD flows, also strong deterioration can be detected. It is clear that a measure of this type can be strongly influenced by the impact of traffic signals on the network. This is true also for other measures such as the outflows. Further, changes in route choices can strongly affect the correspondence of the left-turn movements with the respective measures. The detail of the correspondences between measured and simulated values for the different variables, before and after the O-D estimation process, is reported in the scatter plots of Figure 7. For all variables, except left turning, the correspondence with the measured values is increased by the $\mathrm{O}-\mathrm{D}$ estimation process. The best correspondence is achieved for the link queue: the regression coefficient is $97.7 \%$; the regression slope is near one (1.01); the intercept is close to the origin (-1.19), while the highest improvement is obtained for the link speed (the regression coefficient increases from $86.0 \%$ to $94.7 \%$; the regression slope passes from 1.077 to 1.010 ; the intercept from -5.24 to -0.53$)$.

Summarizing, the solution of the dynamic O-D estimation in combination with different assignment criteria seems 

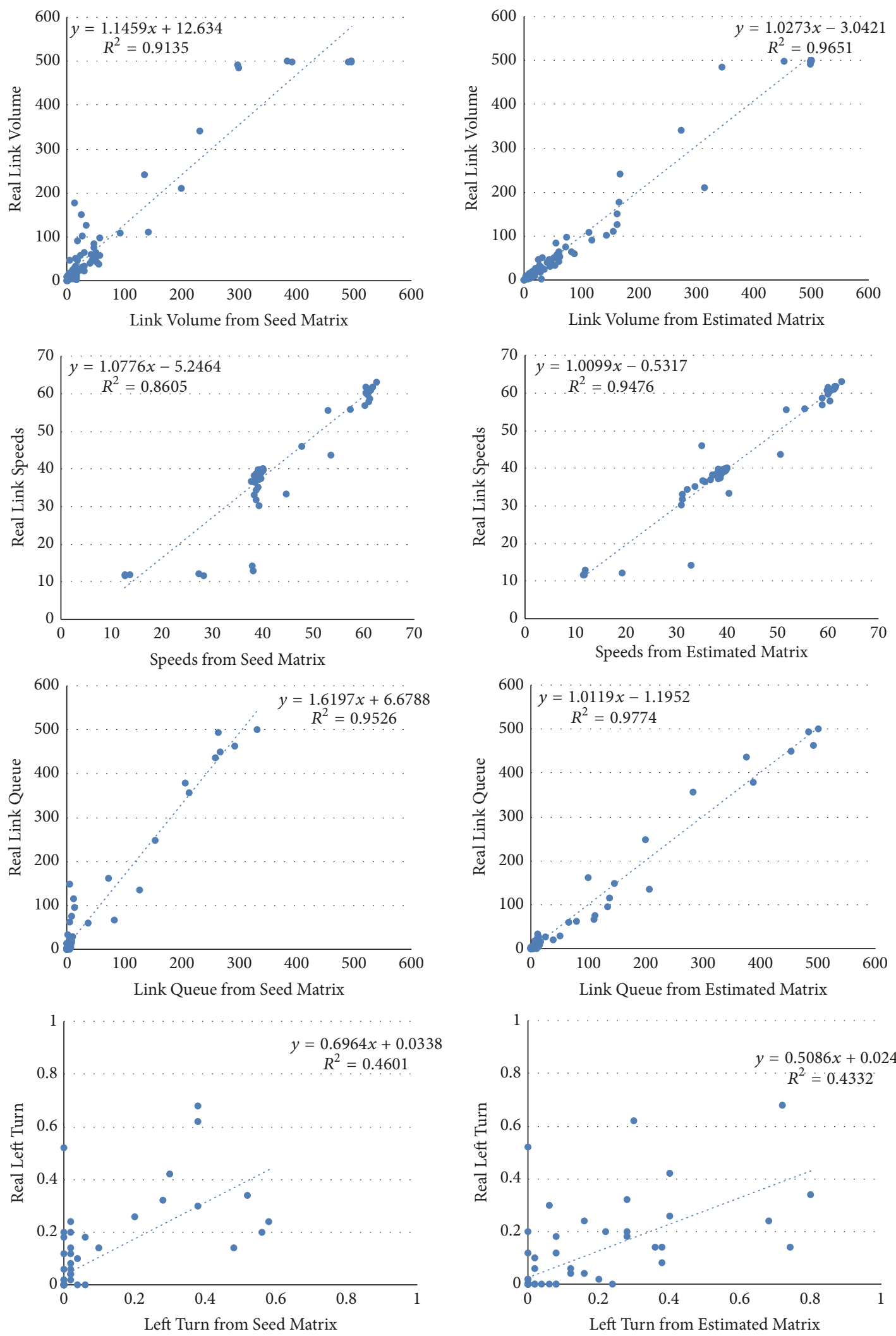

(a)

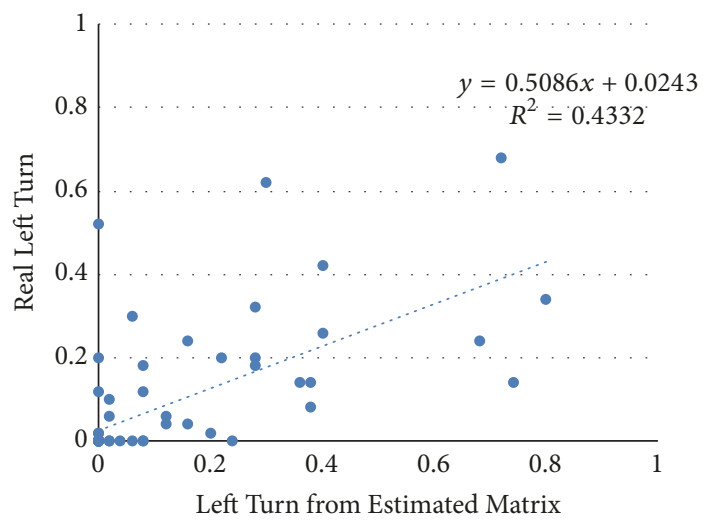

(b)

FiguRE 7: Scatter plots between traffic measures and simulated measures obtained from the seed matrix (a) and from the final estimated matrix (b). 
TABLE 4: Evaluation of the estimated demand as the Euclidean distance from the true O-D matrix and the total number of trips.

\begin{tabular}{|c|c|c|c|}
\hline Reference values & $\begin{array}{c}\text { Euclidean distance } \\
\text { seed-true } \\
459\end{array}$ & $\begin{array}{c}\text { Total trips } \\
\text { seed matrix } \\
12,638\end{array}$ & $\begin{array}{c}\text { Total trips } \\
\text { true matrix } \\
18,900\end{array}$ \\
\hline Experiments & $\begin{array}{c}\text { Euclidean distance } \\
\text { seed-estimated }\end{array}$ & $\begin{array}{c}\text { Euclidean distance } \\
\text { true-estimated }\end{array}$ & $\begin{array}{c}\text { Total trips } \\
\text { estimated demand }\end{array}$ \\
\hline \multicolumn{4}{|l|}{ Set I } \\
\hline Average value & 826.17 & 820.07 & 17,230 \\
\hline St.dev. & 144.04 & 125.74 & 1,714 \\
\hline \multicolumn{4}{|l|}{ Set II } \\
\hline Average value & 817.15 & 786.86 & 17,786 \\
\hline St.dev. & 92.47 & 83.58 & 2.648 \\
\hline \multicolumn{4}{|l|}{ Set IV } \\
\hline Average value & 782.41 & 770.05 & 17,379 \\
\hline St.dev. & 153.42 & 91.24 & 2,641 \\
\hline \multicolumn{4}{|l|}{ Set V } \\
\hline Average value & 437.04 & 548.82 & 15,256 \\
\hline St.dev. & 218.20 & 102.33 & 2,115 \\
\hline \multicolumn{4}{|l|}{ Set VI } \\
\hline Average value & 414.52 & 527.81 & 15,183 \\
\hline St.dev. & 199.09 & 80.78 & 1,496 \\
\hline \multicolumn{4}{|l|}{ Set VII } \\
\hline Average value & 775.20 & 760.26 & 17,403 \\
\hline St.dev. & 212.36 & 152.68 & 2,062 \\
\hline \multicolumn{4}{|l|}{ Set VIII } \\
\hline Average value & 192.48 & 465.05 & 13,307 \\
\hline St.dev. & 63.46 & 12.11 & 466 \\
\hline
\end{tabular}

to underline the efficacy of the adopted algorithm. Also when the QDTA is applied, as an approximation of the DTA, the solutions founded confirm the trend of the other assignment criteria and the results obtained in previous studies: the reproduction of traffic measures is good and when link volumes are considered together with speeds and densities, the improvements are comparable with those obtained in the Dynasmart case by adopting an iterative dynamic assignment approach.

Some problems can be detected in case of instability of route choice to change in the demand, that is to say, when a DNL criteria is followed. This instability can be reduced working on the selected paths, as synthetically made in the experiments with fixed paths, assuming these paths as those selected by road users in real world.

However, the reduction of the $\mathrm{OF}$ and the correct measures reproduction could not be sufficient to appreciate the goodness of the solution. In fact, the aim of the O-D estimation is to find a really reliable demand matrix in order to use it for traffic management and planning applications. For this reason, in the following paragraph, the results have been analyzed as for the capability of O-D estimation method to reproduce the true O-D matrix. This usually cannot be done, unless for laboratory experiments (this is the case), in which the true demand is known.
3.2. Evaluation of the Estimated Demand. Results reported in Table 4 show that the total value of the estimated demand moved in the right direction, passing from the 12,638 trips of the seed matrix to values around 17,000/18,000 trips, the true being 18,900; only when the DNL is at stake (Sets V and $\mathrm{VI}$ ), or when the QDTA is applied, does the O-D estimation procedure stand at lower values of the total demand (about 15,000 trips in the first case and 13,000 trips in the second case).

The distribution in space and time of each O-D has been evaluated measuring the Euclidean Distance (ED) between the estimated matrix and both the true and the seed. Then, these statistics are compared with the reference values of Table 4 . The ED between seed and estimated demand exceeds the value of 770, for Sets I, II, IV, and VII. Moreover, the ED between true and estimated demand is also higher than the ED between seed and true (value of 459). This means that even in the cases where the total value of the estimated demand is very close to the true value, its distribution in space and time is completely different (on average) from the true demand pattern. Then, the estimation method provides different matrices, even very far from the true one, which generate very good matches with the traffic performances measured on the network, as it is possible in such an undetermined problem. This result underlines the importance of 

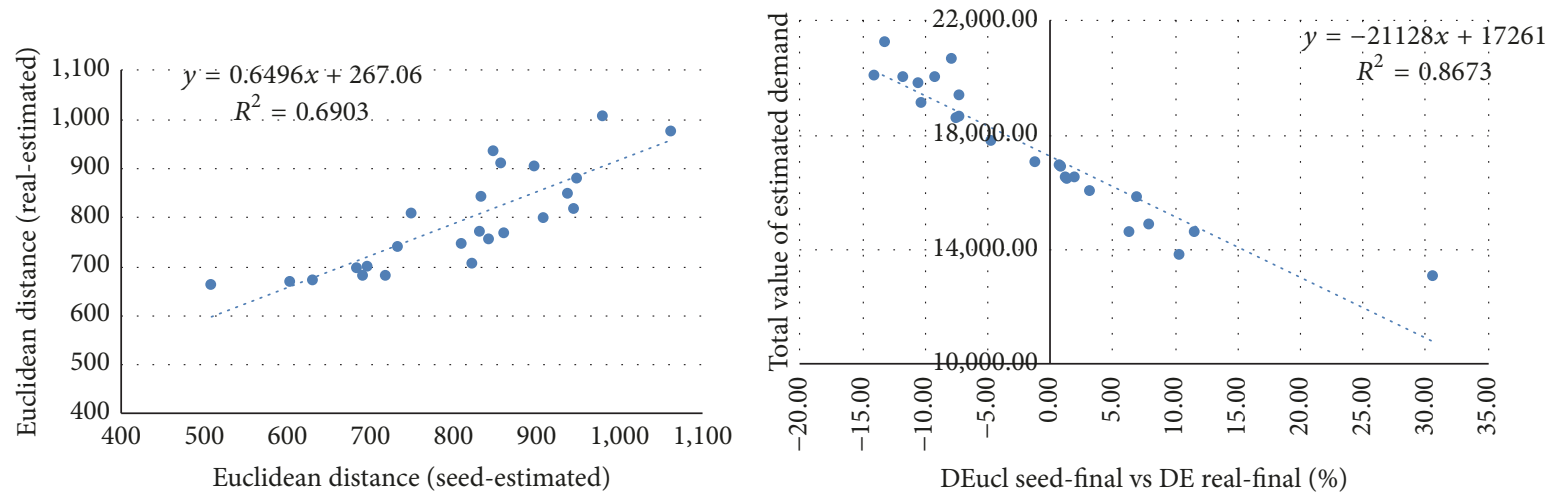

Figure 8: Correlation founded when using DUE, SO, and their combination.

a reliable prior estimate of the $\mathrm{O}-\mathrm{D}$ matrix to ensure that the solution method does not distort the true demand pattern. To go more in depth into this issue, the correlations between the ED seed-estimated and true-estimated have been analyzed and reported in Figure 8; specifically, it seems that the two EDs vary directly proportional to each other; that is, if the prior estimate is poor, also the final is poor. Single results shown in the figure reveal that although on average the estimated matrix is farther from the true than the seed one; in many cases the procedure improves the initial estimation. Moreover, a correlation can be noticed between the two previous EDs and the total value of the estimated demand: with the increase of the total value of the estimated demand, a reduction of the ED true-final with respect to the ED seedfinal is detected (Figure 8).

In the cases of Sets V and VI, as shown in Table 4, the ED between seed and estimated matrices is not higher than the ED between seed and true. Also the ED between true and estimated is quite low (about 500). Then, it seems that some better result has been obtained with respect to the cases where DUE, SO, and their combination are adopted. Effectively, the introduction of fixed DUE paths other than the DNL (Sets $\mathrm{V}$ and VI) has permitted reduction of the noise of the OF (Figure 9).

This noise reduction becomes stronger when the highest O-D flows are intercepted (see Set VI, Table 5). Nonetheless, this results in a greater difficulty while leaving the starting point during the $\mathrm{O}-\mathrm{D}$ estimation and explains the too low value of the total demand (about 15,000 trips).

In case of adoption of the QDTA model (Set VIII), the optimization algorithm does not find the need to move so far from the starting matrix as it does in Dynasmart simulations in order to reproduce the traffic measures. In consequence, the total value of the estimated demand is quite far from the true one (about 13,000 trips with respect to 18,900). However, the $\mathrm{O}-\mathrm{D}$ demand pattern is much closer to the true one than that estimated by applying Dynasmart; in all tests the Euclidean Distance from the true demand is comparable with the corresponding value between starting and true matrix (approximately 459).

In Set VII, the DUE route choice approach, which exhibited the highest convergence capability, has been mixed
TABLE 5: Evaluation of the OF noise (average absolute difference of two following OF values for a mono-dimensional scan between seed and true demand).

\begin{tabular}{lc}
\hline Experiments & OF noise evaluation \\
\hline Set I & 32.19 \\
Set II & 29.18 \\
Set III & 29.62 \\
Set IV & 55.30 \\
Set V & 12.20 \\
Set VI & 10.94 \\
Set VII & 32.33 \\
\hline
\end{tabular}

with the approach that fixes some UE paths with the highest $\mathrm{O}-\mathrm{D}$ flows, which showed the best capacity of mitigating the OF noise. However, this experiment resulted in noise comparable with the basic DUE case (Table 5) and did not introduce significant improvements with respect to matching the true demand (Table 4).

The reason for these results can be explained considering that when adding such fixed DUE paths to the DNL, the route choice variation generated by the dynamic shortest path according to a change in travel demand is strongly limited.

Previous results showed in general a high difficulty in finding the right spatial and temporal distribution of the true demand, even though the total right level can be reached; however there is a need to understand if the problem is in the spatial or in the temporal correlation between O-Ds.

It is worth noting that the test designed was very challenging, since the seed matrix was very different from the true one $(\mathrm{ED}=459$, average difference between $\mathrm{O}$ - $\mathrm{Ds}=$ 25 , maximum difference between $\mathrm{O}-\mathrm{Ds}=60$, and standard deviation between $\mathrm{O}-\mathrm{Ds}=39$ ) and the time resolution for O-D estimation (5 minutes) was much smaller than that usually adopted in dynamic traffic assignment problems (15-20 minutes).

Table 6 reports that the ED values computed summing with respect to the time variable, thus capturing the only space correlation.

In such a case, an improvement with the estimated demand is always obtained and this improvement is higher 


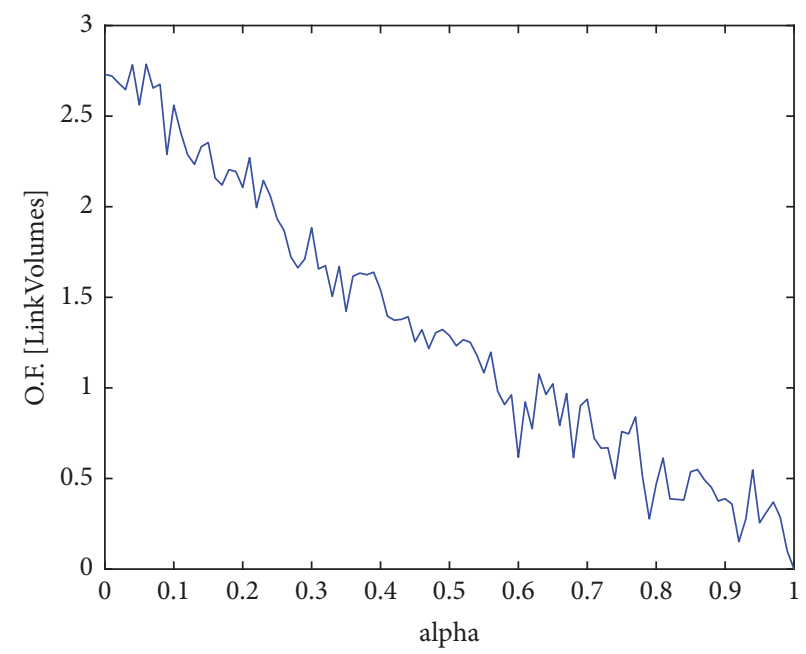

Figure 9: OF1 scan for DNL + fixed UE paths (Set V).

TABLE 6: Evaluation of the estimated demand in terms of O-D distribution in space.

\begin{tabular}{lcccc}
\hline Reference values [ref.] & \multicolumn{3}{c}{$\begin{array}{c}\text { Euclidean distance } \\
\text { seed-true } \\
1,206\end{array}$} \\
Experiments & $\begin{array}{c}\text { Euclidean distance } \\
\text { seed-estimated }\end{array}$ & $\begin{array}{c}\text { Difference with respect } \\
\text { to ref. [\%] }\end{array}$ & $\begin{array}{c}\text { Euclidean distance } \\
\text { true-estimated }\end{array}$ & $\begin{array}{c}\text { Difference with respect } \\
\text { to ref. [\%] }\end{array}$ \\
\hline Set I & 750 & -38 & 1,107 & -8 \\
Set II & 825 & -32 & 1,122 & -7 \\
Set IV & 1,075 & -11 & 1,095 & -10 \\
Set V & 518 & -57 & 1,015 & -16 \\
Set VI & 512 & -58 & 957 & -21 \\
Set VII & 936 & -22 & 924 & -23 \\
Set VIII & 192 & -84 & 1,124 & -7 \\
\hline
\end{tabular}

when fixed paths are adopted: fixed paths are able to simplify the route choice, thus reaching improvement of about $20 \%$ on O-D spatial distribution. In some tests, specifically when they are combined with DNL also improvement of about $30 \%$ has been recorded. The improvement decreases to $10 \%$ if fixed paths are not adopted.

In the QDTA case (Set VIII), the new computed ED values emphasize the difficulty of moving away from the seed matrix and this results in an insufficient ability to reproduce the space distribution of O-Ds (only 7\% of improvement respect to the starting conditions).

To complete the analysis of the results in terms of estimated demand, the ED values are computed aggregating four-time intervals at time by adopting a rolling horizon average method (Table 7), thus resulting in four values covering all the estimated horizon. It is worth mentioning that each experiment tries to estimate a dynamic demand on a full horizon of 35 minutes divided into subintervals of 5 minutes each: thus, the partition of the demand on such a small time interval increases the complexity of the problem. Hence, the estimation needs to be verified on higher time interval, that is, as reported in Table 7.
This last analysis highlights a difficulty in the estimation as time advances, that is, as more demands come through the network and as congestion increases. This is also due to the number of measurements used for the estimation of the demand in any time interval: the demand of the initial time interval which estimated using measurements from the 1st to the 7 th interval, the demand of the second-time interval using measurements from the 2 nd to the 7 th time interval, and so on up to using only one time interval measurements for the estimation of the demand of the last time interval. This difficulty results in worsening the ED for the last two intervals. Again, when fixed paths are introduced, a slight improvement is reported also for these two intervals, where the best result is obtained for Set VI.

At this point, it can be stated that the introduction of information on the followed paths can be a fundamental element to substantially improve the O-D estimation in terms of reproduction of the true demand value and of its distribution in space and time: this is surely suggested by the results obtained for Sets $\mathrm{V}$ and VI but it is also underlined by the difficulties of the $\mathrm{O}-\mathrm{D}$ estimation procedure in reproducing indirectly some measurements as the 
TABLE 7: Evaluation of the estimated demand in terms of O-D aggregated distribution in time.

\begin{tabular}{|c|c|c|c|c|c|}
\hline \multirow{3}{*}{ Reference values [ref.] } & \multirow{3}{*}{ Euclidean distance seed-true } & \multicolumn{4}{|c|}{ Time interval (minutes) } \\
\hline & & $0-20$ & $5-25$ & $10-30$ & $15-35$ \\
\hline & & 700 & 700 & 693 & 682 \\
\hline \multirow{4}{*}{ Set I } & Euclidean distance seed-estimated & 505 & 505 & 617 & 571 \\
\hline & [\% diff. respect to Ref] & {$[-28]$} & {$[-28]$} & {$[-11]$} & {$[-16]$} \\
\hline & Euclidean distance true-estimated & 675 & 662 & 737 & 736 \\
\hline & [\% diff. with respect to ref] & {$[-4]$} & {$[-5]$} & {$[+6]$} & {$[+8]$} \\
\hline \multirow{4}{*}{ Set II } & Euclidean distance seed-estimated & 575 & 526 & 567 & 536 \\
\hline & [\% diff. with respect to ref.] & {$[-18]$} & {$[-25]$} & {$[-18]$} & {$[-21]$} \\
\hline & Euclidean distance true-estimated & 670 & 667 & 751 & 709 \\
\hline & [\% diff. with respect to ref.] & {$[-4]$} & {$[-5]$} & {$[+8]$} & {$[+4]$} \\
\hline \multirow{4}{*}{ Set IV } & Euclidean distance seed-estimated & 737 & 654 & 653 & 656 \\
\hline & [\% diff. with respect to ref.] & {$[+5]$} & {$[-7]$} & {$[-6]$} & {$[-4]$} \\
\hline & Euclidean distance true-estimated & 707 & 663 & 681 & 718 \\
\hline & [\% diff. with respect to ref.] & {$[+1]$} & {$[-5]$} & {$[-2]$} & {$[+5]$} \\
\hline \multirow{4}{*}{ Set V } & Euclidean distance seed-estimated & 391 & 342 & 337 & 351 \\
\hline & [\% diff. with respect to ref.] & {$[-44]$} & {$[-51]$} & {$[-51]$} & {$[-49]$} \\
\hline & Euclidean distance true-estimated & 583 & 593 & 646 & 648 \\
\hline & [\% diff. with respect to ref.] & {$[-17]$} & {$[-15]$} & {$[-7]$} & {$[-5]$} \\
\hline \multirow{4}{*}{ Set VI } & Euclidean distance seed-estimated & 376 & 384 & 332 & 286 \\
\hline & [\% diff. with respect to ref.] & {$[-46]$} & {$[-45]$} & {$[-52]$} & {$[-58]$} \\
\hline & Euclidean distance true-estimated & 552 & 590 & 562 & 568 \\
\hline & [\% diff. with respect to ref.] & {$[-21]$} & {$[-16]$} & {$[-19]$} & {$[-17]$} \\
\hline \multirow{4}{*}{ Set VII } & Euclidean distance seed-estimated & 642 & 627 & 576 & 579 \\
\hline & [\% diff. with respect to ref.] & {$[-8]$} & {$[-10]$} & {$[-17]$} & {$[-15]$} \\
\hline & Euclidean distance true-estimated & 600 & 555 & 549 & 577 \\
\hline & [\% diff. with respect to ref.] & {$[-14]$} & {$[-21]$} & {$[-21]$} & {$[-15]$} \\
\hline \multirow{4}{*}{ Set VIII } & Euclidean distance seed-estimated & 737 & 654 & 653 & 656 \\
\hline & [\% diff. with respect to ref.] & {$[+5]$} & {$[-7]$} & {$[-6]$} & {$[-4]$} \\
\hline & Euclidean distance true-estimated & 638 & 684 & 676 & 667 \\
\hline & [\% diff. with respect to ref.] & {$[-9]$} & {$[-2]$} & {$[-2]$} & {$[-2]$} \\
\hline
\end{tabular}

left-turn movements. As already mentioned, the left-turn movements are strongly related to the route choices. Since many matrices can reproduce quite perfectly link measures as volumes and speeds and also link queues and outflows, without being the true demand, an effective way to reproduce the true conditions on the traffic networks, even for shortterm planning and management operations, is to be able to reproduce the correct path choices.

For this reason, some final experiments have been conducted adding path choices information during the estimation in terms of O-D travel times inside the OF.

\subsection{Use of Path Choices Information during the Estimation} Procedure. Recent ICT enhancements provide increasing deployment of identification sensors. In addition to traditional monitoring devices that collect information on the whole traffic stream at fixed locations, identification sensors allow tracking individual vehicles both at fixed locations on the road and on the network ubiquitously. Examples of fixed identification sensors are image recognition cameras and radio sensors that can capture public information from bluetooth and Wi-Fi devices on board of vehicles. Ubiquitous monitoring consists of sampling vehicles equipped with GPS location devices and mobile cellular data transmitters along their paths. The latter form of monitoring is particularly efficient, because it uses the same devices to provide information to drivers and get information by them, is very effective, and provides information on origin-destination of individual trips as well as on the routes travelled by the drivers.

This information is fundamental to get an accurate knowledge of travel demand and achieve reliable, wellcalibrated road traffic models [43].

Today, Floating Car Data (FCD) provide a huge amount of true-time updated datasets on vehicle positions and speeds, 
TABLE 8: Evaluation of the estimated demand as the Euclidean distance from the true O-D matrix and the total number of trips (adding O-D travel times).

\begin{tabular}{|c|c|c|c|}
\hline Reference values & $\begin{array}{c}\text { Euclidean distance } \\
\text { seed-true } \\
459\end{array}$ & $\begin{array}{c}\text { Total trips } \\
\text { seed matrix } \\
12,638\end{array}$ & $\begin{array}{c}\text { Total trips } \\
\text { true matrix } \\
18,900\end{array}$ \\
\hline Experiments & $\begin{array}{l}\text { Euclidean distance } \\
\text { seed-estimated }\end{array}$ & $\begin{array}{l}\text { Euclidean distance } \\
\text { true-estimated }\end{array}$ & $\begin{array}{c}\text { Total trips } \\
\text { estimated demand }\end{array}$ \\
\hline \multicolumn{4}{|l|}{ Set I } \\
\hline Average value & 654.62 & 578.86 & 18,635 \\
\hline St.dev. & 158.46 & 70.58 & 2,662 \\
\hline \multicolumn{4}{|l|}{ Set VI } \\
\hline Average value & 566.22 & 539.25 & 17,335 \\
\hline St.dev. & 16.68 & 17.68 & 155 \\
\hline \multicolumn{4}{|l|}{ Set VIII } \\
\hline Average value & 211.00 & 508.69 & 12,845 \\
\hline St.dev. & 75.87 & 28.37 & 147 \\
\hline
\end{tabular}

TABLE 9: Evaluation of the estimated demand in terms of O-D distribution in space (adding O-D travel times).

\begin{tabular}{|c|c|c|c|c|}
\hline $\begin{array}{l}\text { Reference values } \\
\text { [ref.] }\end{array}$ & & $\begin{array}{c}\text { Euclidean distance } \\
\text { seed-true } \\
1,206\end{array}$ & & \\
\hline Experiments & $\begin{array}{c}\text { Euclidean distance } \\
\text { seed-estimated }\end{array}$ & $\begin{array}{l}\text { Difference with } \\
\text { respect to ref. [\%] }\end{array}$ & $\begin{array}{c}\text { Euclidean distance } \\
\text { true-estimated }\end{array}$ & $\begin{array}{l}\text { Difference with } \\
\text { respect to ref. [\%] }\end{array}$ \\
\hline Set I & 893 & -26 & 838 & -31 \\
\hline Set VI & 1,003 & -17 & 891 & -26 \\
\hline Set VIII & 313 & -74 & 1,184 & -2 \\
\hline
\end{tabular}

which allow estimating both travel times and routes followed by vehicles tracked. They should be exploited to improve demand estimation and modeling assumptions.

In this last section, O-D travel times by FCD have been added inside the OF (1) next to link measurements.

These travel times have been practically obtained as a result of the assignment of the true demand (with different assignment criteria), as already done for the collected link measurements in previous experiments.

The following sets have been analyzed through adding the O-D travel times to (i) Set I, since it is based on DUE criterion, which theoretically represents the real user behaviour in case of congested conditions; (ii) Set VI, where promising results have been obtained in the previous experiments including fixed paths; (iii) Set VIII, in order to continue the investigation of approximating the DTA with the QDTA.

Tables 8, 9, and 10 report the analysis of the estimated demand values for each set.

Previous experiments adopting the DUE (Set I), without adding information on travel times, demonstrated the ability to obtain the right level of total demand, but problems in terms of reproducing its temporal distribution were detected. The introduction of O-D travel times (Table 8, Set I), other than the level of total demand that remains comparable with the true value, generated a significant reduction of the ED (from 820 , Table 4, to 578, Table 8), which is comparable with the ED values obtained when fixed paths are adopted.
A detailed analysis of the results in Set I derives the fact that the adoption of the new information on travel times in the objective function enables the estimation procedure to reproduce the correct path choices. In fact, not only improvements on link measures are as high as those already obtained in previous tests (without O-D travel times) for volumes, speeds, and densities (higher than 95\%), but, in addition, outflows ( $80 \%$ of improvement), queue length (99\%) and, more importantly, left-turn movements $(73 \%)$ are now properly estimated. Such occurrence indicates that with O-D travel time information the estimation procedure can simulate the correct traffic regime and the correct route choices. This is demonstrated also by the values reported in Tables 9 and 10, Set I reporting a substantial improvement both in the spatial and temporal distribution of the O-D variables, with respect to the "no travel times" case. This is verified only when DUE criteria are adopted. These results are in accordance with other recent research contributions [30] which demonstrated that link measurements alone are not able to define the real traffic pattern on a road network. Instead, if information on route choices on the road network is available (and this is becoming increasingly easier with the large development of advanced monitoring systems), this may guarantee a very refined solution in terms of estimated demand.

In case of Set VI, the O-D travel times allowed us to obtain the correct quantity of total demand with respect 
TABLE 10: Evaluation of the estimated demand in terms of $\mathrm{O}-\mathrm{D}$ aggregated distribution in time (adding O-D travel times).

\begin{tabular}{|c|c|c|c|c|c|}
\hline $\begin{array}{l}\text { Reference values } \\
\text { [ref.] }\end{array}$ & Euclidean distance seed-true & 700 & 700 & 693 & 682 \\
\hline \multirow{4}{*}{ Set I } & Euclidean distance seed-estimated & 607 & 593 & 481 & 516 \\
\hline & [\% diff. with respect to ref.] & {$[-13]$} & {$[-15]$} & {$[-31]$} & {$[-24]$} \\
\hline & Euclidean distance true-estimated & 545 & 519 & 515 & 576 \\
\hline & [\% diff. with respect to ref.] & {$[-22]$} & {$[-26]$} & {$[-26]$} & {$[-16]$} \\
\hline \multirow{4}{*}{ Set VI } & Euclidean distance seed-estimated & 596 & 607 & 637 & 627 \\
\hline & [\% diff. with respect to ref.] & {$[-15]$} & {$[-13]$} & {$[-8]$} & {$[-8]$} \\
\hline & Euclidean distance true-estimated & 557 & 580 & 569 & 544 \\
\hline & [\% diff. with respect to ref.] & {$[-20]$} & {$[-17]$} & {$[-18]$} & {$[-20]$} \\
\hline \multirow{4}{*}{ Set VIII } & Euclidean distance seed-estimated & 259 & 254 & 178 & 171 \\
\hline & [\% diff. with respect to ref.] & {$[-63]$} & {$[-64]$} & {$[-74]$} & {$[-75]$} \\
\hline & Euclidean distance true-estimated & 716 & 691 & 676 & 649 \\
\hline & [\% diff. with respect to ref.] & {$[+2]$} & {$[-1]$} & {$[-2]$} & {$[-5]$} \\
\hline
\end{tabular}

to the underestimation of the previous tests. Instead, the ED between real and estimated demand is almost the same (compare Set VI, Tables 8 and 4). In previous experiments without O-D travel times, the right total level of demand has been obtained only when DUE or SO criteria have been used. Improvements can be recorded not only in terms of total demand value, but also in terms of spatial distribution (26\% of improvement with respect to the starting point, Table 9). Negligible is the improvement of the temporal distribution which is very close to what was already obtained without travel times (Table 10). This proves that adding $\mathrm{O}-\mathrm{D}$ travel times to Set VI, where path information was somehow already included (fixed paths), enforces the estimation procedure in reproducing the right demand level rather than refining the simulated route choices.

Finally, adopting the QDTA (Set VIII), the O-D travel times do not seem to add further information during the estimation procedure. Results are quite similar to those obtained without travel times; indeed it seems to get worse as the solution moves away from the seed matrix.

\section{Conclusions and Further Research}

The paper has dealt with the impact of introducing different sources of information and different route choice criteria in the O-D demand estimation problem, solved by the Simultaneous Perturbation Stochastic Approximation with Asymmetric Design and Polynomial Interpolation (SPSA AD-PI) method. A systematic analysis, performed on a test network, has showed that the estimation procedure is characterized by good or excellent convergence properties in all the cases examined other than when Dynamic Network Loading (DNL) method is adopted for performing assignment phase; in this case, the assignment criteria prevent the estimation algorithm from converging to a solution.

Nevertheless, the estimated O-D matrix in most cases is not satisfactory when properly compared to the true solution and may be even farther from it than the initial seed O-D matrix.
This is not surprising, provided that the problem is highly undetermined and highly nonconvex and that the distance from the initial matrix was not included into the objective function nor the was search limited by any constraint. This result highlights the importance of including a good initial estimate of the $\mathrm{O}-\mathrm{D}$ matrix in the estimation process.

Among the different variables examined, the most important contribution to demand estimation has been, in most cases, achieved by using measurements of traffic counts, link speed, and queue length.

Apart from the various performance measures, the analysis has shown that the route choice is a main factor, since it affects the spatial correlation between link performances and O-D flows.

Indeed, when some routes are fixed, the performances of the method are improved. Specifically, better results are obtained when only some routes are fixed for some given $\mathrm{O}-\mathrm{D}$ pairs and the other routes can change over time, as in the case in which DNL is applied. Worse results are obtained when the other routes can change over both the time and the space (as in the case in which DUE is applied).

The paper has dealt with the research line concerning the way to improve the effectiveness of the estimation method introducing information on route choices observed from Floating Car Data (FCD) collected in the field; this research line, still ongoing, seems to be a promising approach. Achieving direct information on drivers' route choices and the related experienced route travel times opens promising perspectives also for a joint calibration of the O-D demand and the traffic assignment model and is expected to provide new insights into the real existence of dynamic equilibria [44]. This is confirmed by experiments carried out adding $\mathrm{O}-\mathrm{D}$ travel times measurements in the estimation procedure that demonstrated the importance and the need to provide information on paths and not only on links, in order to properly estimate the temporal demand; besides, results indicate that this type of information enables the procedure to simulate the correct traffic conditions on the network and the real user route choices; this is verified mainly when the 
DUE criterion is applied, since route travel times directly affect link performances; this is not the case of DNL where the reproduction of the correct route choices is not assured. The approximation of DTA with QDTA, although capable of reproducing the common traffic measures adopted in the O-D estimation problem (mainly flows and speeds), does not seem currently able to exploit additional information on paths. In fact, QDTA assumes pretrip information and performs a probabilistic network loading according to the travel times computed in the previous time interval, so that the relationship between $\mathrm{O}-\mathrm{D}$ flows and route travel times is less straightforward than in DUE model. The relevance of this research line is confirmed by recent research efforts that evaluate the effectiveness of information at network level, such as travel times and route choice probabilities derived from FCD, in the dynamic demand estimation process for real network cases [30].

A second research line, which will require further study with respect to the results here obtained, concerns the suitable degree of approximation for the dynamic O-D estimation problem. While the availability of many sources of information makes it possible to better appreciate the contribution of sophisticated models, it also enlarges the problem of dimensions and requires longer computation times. On the other hand, approximate traffic assignment models, such as quasi-dynamic assignment models, reduce both the calibration efforts and the computation time and might result in more advantageous for applications to timedependent $\mathrm{O}-\mathrm{D}$ estimation problems, where approximate algorithms are introduced to solve the problem on real-size networks. A question worth of investigation is the degree of approximation that can be introduced in the different steps of the estimation method, that is, the SPSA algorithm, which provides some numerical approximation of the gradient method; QDTA model, which approximates the dynamic model by steady-state intervals and applies approximate performance functions; a priori hypotheses on the time-space structure of the demand, assuming some correlations as fixed, as done by Cascetta et al. [27].

Finally, a third research line, which refers to the contents analyzed in this paper, concerns the structure of the objective function and specifically the investigation of the performances of SPSA AD-PI solution method when applied to the vector formulation recently introduced by Lu et al. [12], which showed to improve the efficiency of traditional SPSA method significantly.

\section{Conflicts of Interest}

The authors declare that they have no conflicts of interest.

\section{References}

[1] E. Cipriani and M. Nigro, "Dynamic travel demand estimation and prediction methods," Intelligent Transport Systems (ITS): Past, Present and Future Directions, pp. 231-250, 2017.

[2] E. Cascetta, D. Inaudi, and G. Marquis, "Dynamic estimators of origin-destination matrices using traffic counts," Transportation Science, vol. 27, no. 4, pp. 363-373, 1993.
[3] V. Marzano, A. Papola, and F. Simonelli, "Limits and perspectives of effective O-D matrix correction using traffic counts," Transportation Research Part C: Emerging Technologies, vol. 17, no. 2, pp. 120-132, 2009.

[4] C. Antoniou, J. Barceló, M. Breen et al., "Towards a generic benchmarking platform for origin-destination flows estimation/updating algorithms: Design, demonstration and validation," Transportation Research Part C: Emerging Technologies, vol. 66, pp. 79-98, 2016.

[5] H. Yang, "Heuristic algorithms for the bilevel origin-destination matrix estimation problem," Transportation Research Part B: Methodological, vol. 29, no. 4, pp. 231-242, 1995.

[6] R. Balakrishna and H. N. Koutsopoulos, "Incorporating withinday transitions in simultaneous offline estimation of dynamic origin-destination flows without assignment matrices," Transportation Research Record, no. 2085, pp. 31-38, 2008.

[7] E. Cipriani, M. Florian, M. Mahut, and M. Nigro, "A gradient approximation approach for adjusting temporal origindestination matrices," Transportation Research Part C: Emerging Technologies, vol. 19, no. 2, pp. 270-282, 2011.

[8] X. Zhou, C. Lu, and K. Zhang, Dynamic Origin-Destination Demand Flow Estimation Utilizing Heterogeneous data sources under Congested Traffic Conditions, Accessed March 2014, Available online at: http://onlinepubs.trb.org/onlinepubs/ conferences/2012/4thITM/Papers-A/0117-000097.pdf, 2012.

[9] T. Djukic, G. Flotterod, H. van Lint, and S. Hoogendoorn, "Efficient real time OD matrix estimation based on Principal Component Analysis," in Proceedings of the 2012 15th International IEEE Conference on Intelligent Transportation Systems - (ITSC 2012), vol. 2, pp. 115-121, Anchorage, Alaska, USA, September 2012.

[10] G. Cantelmo, F. Viti, C. Tampêre, E. Cipriani, and M. Nigro, "Two-Step Approach for Correction of Seed Matrix in Dynamic Demand Estimation," Transportation Research Record, vol. 2466, pp. 125-133, 2014.

[11] G. Cantelmo, E. Cipriani, A. Gemma, and M. Nigro, "An adaptive Bi-level gradient procedure for the estimation of dynamic traffic demand," IEEE Transactions on Intelligent Transportation Systems, vol. 15, no. 3, pp. 1348-1361, 2014.

[12] L. Lu, Y. Xu, C. Antoniou, and M. Ben-Akiva, "An enhanced SPSA algorithm for the calibration of Dynamic Traffic Assignment models", Transportation Research Part C: Emerging Technologies, vol. 51, pp. 149-166, 2015.

[13] R. Balakrishna, Off-line calibration of dynamic traffic assignment models [Ph.D. thesis], Massachusetts Institute of Technology, 2006.

[14] M. P. Dixon and L. R. Rilett, "Real-time OD estimation using automatic vehicle identification and traffic count data," Computer-Aided Civil and Infrastructure Engineering, vol. 17, no. 1, pp. 7-21, 2002.

[15] S. Eisenman and G. F. List, "Using probe data to estimate OD matrices," in Proceedings of the 7th International IEEE Conference on Intelligent Transportation Systems, pp. 291-296, Washington, DC, USA, 2004.

[16] C. Antoniou, M. Ben-Akiva, and H. N. Koutsopoulos, "Incorporating automated vehicle identification data into origindestination estimation," Transportation Research Record, no. 1882, pp. 37-44, 2004.

[17] X. Zhou and H. S. Mahmassani, "Dynamic origin-destination demand estimation using automatic vehicle identification data," IEEE Transactions on Intelligent Transportation Systems, vol. 7, no. 1, pp. 105-114, 2006. 
[18] N. Caceres, J. P. Wideberg, and F. G. Benitez, "Deriving origindestination data from a mobile phone network," IET Intelligent Transport Systems, vol. 1, no. 1, pp. 15-26, 2007.

[19] J. Barcel, L. Montero, M. Bullejos, O. Serch, C. Carmona, and J. Barceló, Dynamic OD Matrix Estimation Exploiting Bluetooth Data in Urban Networks, Recent Researches in Automatic Control and Electronics, 2012, ISBN: 978-1-61804-080-0.

[20] E. Mitsakis, J. M. Salanova, E. Chrysohoou, and G. Aifadopoulou, "A robust method for real-time estimation of travel times for dense urban road networks using point-topoint detectors," in Proceedings of the 92nd Annual Meeting in Transportation Research Board, TRB, 2013.

[21] J. Kim, F. Kurauchi, N. Uno, T. Hagihara, and T. Daito, "Using electronic toll collection data to understand traffic demand," Journal of Intelligent Transportation Systems, vol. 18, no. 2, pp. 190-203, 2014.

[22] D. Iannò and M. N. Postorino, "A generation constrained approach for the estimation of O/D trip matrices from traffic counts," in Proceedings of the European Transport Conference 2002, Homerton College, Cambridge, England, 2002.

[23] E. Cipriani, M. Florian, M. Mahut, and M. Nigro, "Investigating the efficiency of a gradient approximation approach for solution of dynamic demand estimation problem," New Developments In Transport Planning - Advances in Dynamic Traffic Assignment, 2010.

[24] E. Cipriani, M. Nigro, G. Fusco, and C. Colombaroni, "Effectiveness of link and path information on simultaneous adjustment of dynamic O-D demand matrix," European Transport Research Review, vol. 6, no. 2, pp. 139-148, 2014.

[25] R. Frederix, F. Viti, R. Corthout, and C. M. J. Tampère, "New gradient approximation method for dynamic origindestination matrix estimation on congested networks," Transportation Research Record, vol. 2263, pp. 19-25, 2011.

[26] C. C. Lu, X. Zhou, and K. Zhang, "Dynamic origin-destination demand flow estimation under congested traffic conditions," Transportation Research Part C: Emerging Technologies, vol. 34, pp. 16-37, 2013.

[27] E. Cascetta, A. Papola, V. Marzano, F. Simonelli, and I. Vitiello, "Quasi-dynamic estimation of o-d flows from traffic counts: formulation, statistical validation and performance analysis on real data," Transportation Research Part B: Methodological, vol. 55, pp. 171-187, 2013.

[28] E. Cipriani, A. Gemma, M. Nigro, C. Colombaroni, and G. Fusco, "Reliability of SPSA AD-PI method for dynamic demand estimation: a case of study," in Proceedings of the 11th International Congress on Advanced in Civil Engineering, pp. 21-25, Istanbul, Turkey, 2014.

[29] G. Fusco, C. Colombaroni, A. Gemma, and S. Lo Sardo, "A quasi-dynamic traffic assignment model for large congested urban road networ," Mathematical Models and Methods in Applied Sciences, vol. 7, no. 4, pp. 341-349, 2013.

[30] M. Nigro, E. Cipriani, and A. del Giudice, "Exploiting floating car data for time-dependent OriginDestination matrices estimation," Journal of Intelligent Transportation Systems: Technology, Planning, and Operations, pp. 10-1080, 2017.

[31] S. Carrese, E. Cipriani, L. Mannini, and M. Nigro, "Dynamic demand estimation and prediction for traffic urban networks adopting new data sources," Transportation Research Part C: Emerging Technologies, vol. 81, pp. 83-98, 2017.

[32] G. Fusco, A. Bracci, T. Caligiuri, C. Colombaroni, and N. Isaenko, "Experimental analyses and clustering of travel choice behaviours by floating car big data in a large urban area," IET Intelligent Transport Systems, vol. 12, no. 4, pp. 270-278, 2018.

[33] G.-L. Chang, H. S. Mahmassani, and R. Herman, "Macroparticle traffic simulation model to investigate peak-period commuter decision dynamics," Transportation Research Record, vol. 1005, pp. 107-121, 1985.

[34] H. S. Mahmassani and G.-L. Chang, "Experiments with departure time choice dynamics of urban commuters," Transportation Research Part B: Methodological, vol. 20, no. 4, pp. 297-320, 1986.

[35] H. S. Mahmassani, G.-L. Chang, and R. Herman, "Individual decisions and collective effects in simulated traffic system," Transportation Science, vol. 20, no. 4, pp. 258-271, 1986.

[36] T. Y. Hu, R. W. Rothery, and H. Mahmassani, DYNASMART: DYnamic Network Assignment-Simulation Model for Advanced Road Telematics, Working Paper DTFH61-90-C00074-TWP1, Center for Transportation, The University of Texas at Austin, January, 1992.

[37] H. S. Mahmassani, T. Hu, and R. Jayakrishnan, "Dynamic traffic assignment and simulation for advanced network informatics," in Proceedings of the Second International Capri Seminar on Urban Traffic Networks, Capri, Italy, 1992.

[38] R. Frederix, F. Viti, and C. M. J. Tampère, "Dynamic origindestination estimation in congested networks: theoretical findings and implications in practice," Transportmetrica A: Transport Science, vol. 9, no. 6, pp. 494-513, 2013.

[39] H. S. Mahmassani, H. Sbayti, and X. Zhou, DYNASMART-P Version 1.0 User's Guide. Maryland Transportation Initiative, College Park, Maryland, 137, 2004.

[40] A. Abdelfatah, Combined Time-Dependent Signal Timing and System Optimal Dynamic Traffic Assignment in Congested Vehicular Traffic Networks [Ph.D. thesis], The University of Texas, Austin, Tex, USA, 1999.

[41] J. C. Spall, "An overview of the simultaneous perturbation method for efficient optimization," Johns Hopkins APL Technical Digest, vol. 19, no. 4, pp. 482-492, 1998.

[42] J. C. Spall, "Implementation of the simultaneous perturbation algorithm for stochastic optimization," IEEE Transactions on Aerospace and Electronic Systems, vol. 34, no. 3, pp. 817-823, 1998.

[43] G. Fusco, C. Colombaroni, L. Comelli, and N. Isaenko, "Short-term traffic predictions on large urban traffic networks: Applications of network-based machine learning models and dynamic traffic assignment models," in Proceedings of the International Conference on Models and Technologies for Intelligent Transportation Systems, MT-ITS 2015, pp. 93-101, Budapest, Hungary, June 2015.

[44] G. Fusco, C. Colombaroni, and N. Isaenko, "Dynamic traveler information systems," Intelligent Transport Systems (ITS): Past, Present and Future Directions, pp. 265-314, 2017. 


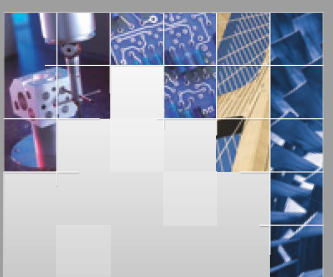

\section{Enfincering}
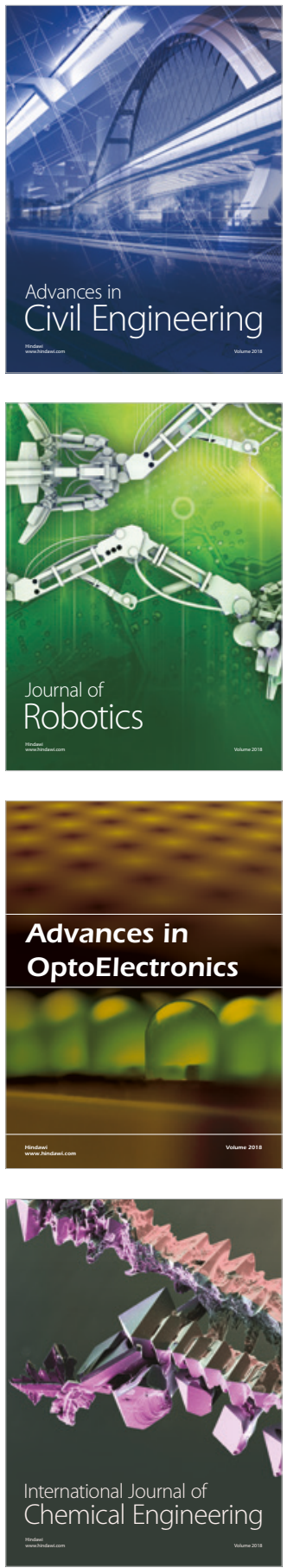

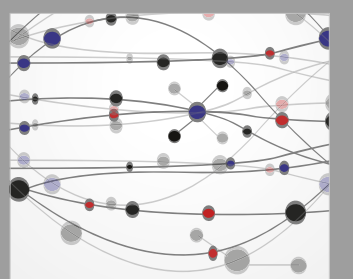

\section{Rotating \\ Machinery}

The Scientific World Journal

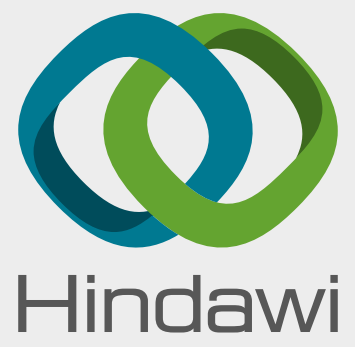

Submit your manuscripts at

www.hindawi.com
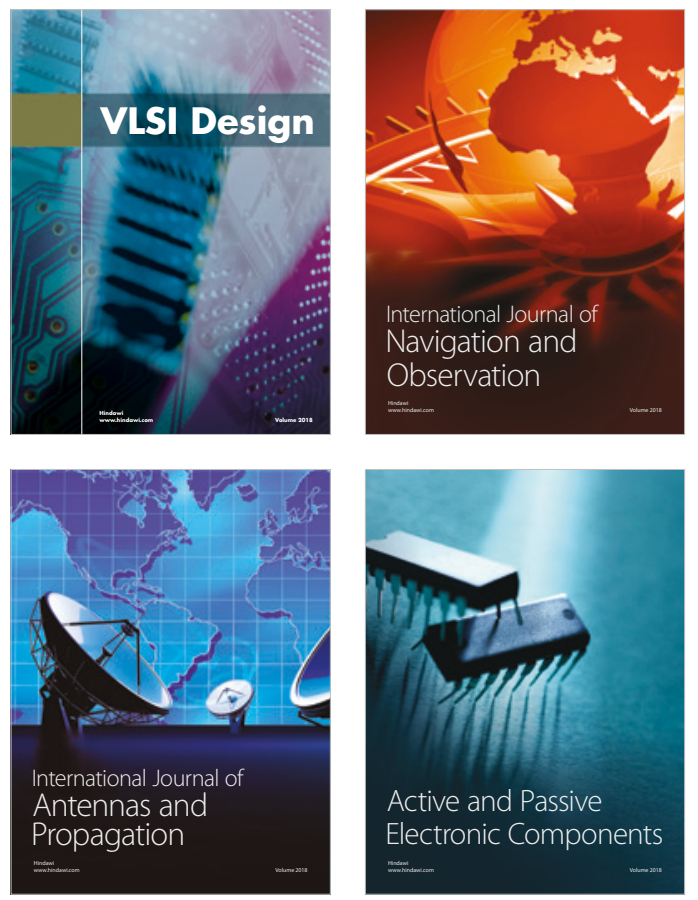
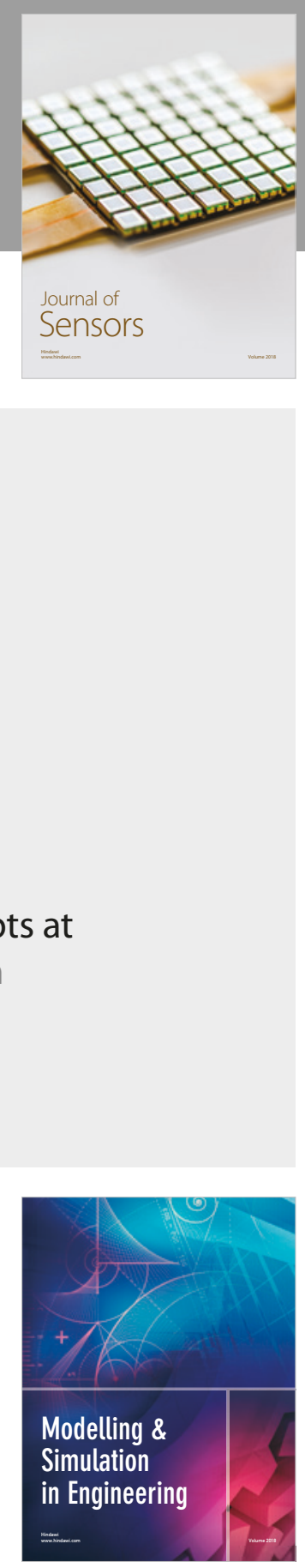

\section{Advances \\ Multimedia}
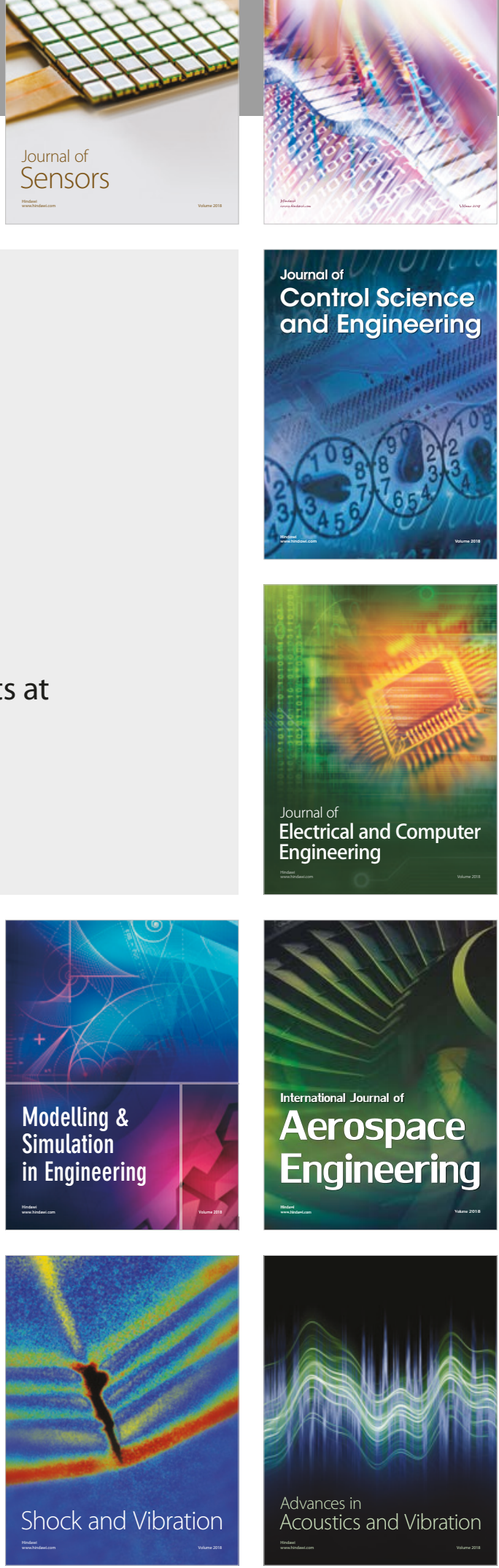